\title{
Cell-derived microparticles and the lung
}

\author{
Dario Nieri ${ }^{1,2}$, Tommaso Neri ${ }^{1,2}$, Silvia Petrini ${ }^{1}$, Barbara Vagaggini ${ }^{1}$, \\ Pierluigi Paggiaro ${ }^{1}$ and Alessandro Celi ${ }^{1}$
}

Affiliations: ${ }^{1}$ Laboratorio di Biologia Cellulare Respiratoria, SVD Fisiopatologia Respiratoria e Riabilitazione, Dipartimento di Patologia Chirurgica, Medica, Molecolare e dell'Area Critica, University of Pisa, Pisa, Italy. ${ }^{2}$ Both authors contributed equally.

Correspondence: Alessandro Celi, Università di Pisa, Dipartimento Cardiotoracico e Vascolare, Via Paradisa 2. 56124, Pisa, Italy. E-mail: alessandro.celiamed.unipi.it

ABSTRACT Cell-derived microparticles are small $(0.1-1 \mu \mathrm{m})$ vesicles shed by most eukaryotic cells upon activation or during apoptosis. Microparticles carry on their surface, and enclose within their cytoplasm, molecules derived from the parental cell, including proteins, DNA, RNA, microRNA and phospholipids. Microparticles are now considered functional units that represent a disseminated storage pool of bioactive effectors and participate both in the maintenance of homeostasis and in the pathogenesis of diseases. The mechanisms involved in microparticle generation include intracellular calcium mobilisation, cytoskeleton rearrangement, kinase phosphorylation and activation of the nuclear factor- $\kappa \mathrm{B}$. The role of microparticles in blood coagulation and inflammation, including airway inflammation, is well established in in vitro and animal models. The role of microparticles in human pulmonary diseases, both as pathogenic determinants and biomarkers, is being actively investigated. Microparticles of endothelial origin, suggestive of apoptosis, have been demonstrated in the peripheral blood of patients with emphysema, lending support to the hypothesis that endothelial dysfunction and apoptosis are involved in the pathogenesis of the disease and represent a link with cardiovascular comorbidities. Microparticles also have potential roles in patients with asthma, diffuse parenchymal lung disease, thromboembolism, lung cancer and pulmonary arterial hypertension.

@ERSpublications

Microparticles are potential biomarkers and targets for therapeutic interventions in respiratory medicine http://ow.ly/ZTCp6

\section{Introduction}

Virtually all eukaryotic cells shed submicron vesicles constitutively, upon activation or during apoptosis. While their existence has been known for a long time, these structures were initially considered laboratory artefacts or, at most, cell debris devoid of physiological significance. Only relatively recently have researchers begun to appreciate their potential roles in physiology and pathophysiology, and their involvement in processes as diverse as, for example, blood coagulation, inflammation, intercellular signalling and tumour cell growth. Cell-derived vesicles may vary in size, composition and mechanisms of generation, and depending on these characteristics they are usually called exosomes, microparticles (or ectosomes) and apoptotic bodies; however, their precise identification and characterisation has proven difficult, and a shared nomenclature for the different types of vesicles is still lacking.

In this review we will first discuss some basic aspects of microparticle function and generation and then describe the roles of these vesicles as potential mediators and biomarkers in respiratory diseases. While we will try to focus on the structures usually referred to as microparticles, it is not possible to avoid that some of the data presented here are in fact related to other types of vesicles.

Received: Feb 092016 | Accepted after revision: March 192016

Conflict of interest: Disclosures can be found alongside the online version of this article at err.ersjournals.com

Provenance: Submitted article, peer reviewed.

Copyright CERS 2016. ERR articles are open access and distributed under the terms of the Creative Commons Attribution Non-Commercial Licence 4.0. 

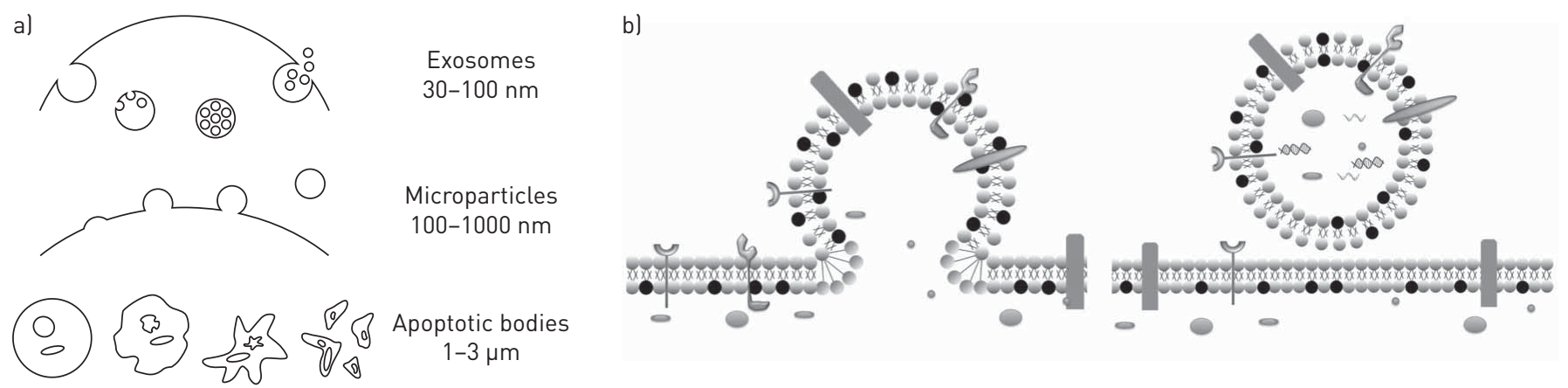

FIGURE 1 Schematic representations of a) the three types of extracellular vesicles and b) microparticle generation in more detail.

\section{Differential characteristics of extracellular vesicles}

Cell-derived vesicles can be defined as spherical particles enclosed by a phospholipid bilayer [1]. The dimensions of these structures vary and represent one of the characteristics that distinguish the three types of vesicles previously mentioned: exosomes typically range in size between 30 and $100 \mathrm{~nm}$, microparticles range between 100 and $1000 \mathrm{~nm}$, while apoptotic bodies are larger, with diameters between 1 and $3 \mu \mathrm{m}$ [2]. Exosomes are generated by inward budding of the membrane that leads to the generation of multivesicular bodies; these structures then fuse with the cell membrane and are released as exosomes [3]. Apoptotic bodies are generated upon cell shrinkage and apoptotic death [3]. Microparticles, which as previously stated represent the focus of this review, are formed by the outward blebbing of the plasma membrane with subsequent release after proteolytic cleavage of the cytoskeleton [4]. Figure 1a shows the mechanisms of formation of these three types of vesicles, while figure $1 \mathrm{~b}$ represents a more detailed schematic of microparticle generation.

\section{Historical perspective: microparticles as "platelet dust"}

In 1955, O’BRIEN [5] described "platelet-like activity" in normal human serum. 12 years later, Wolf [6] came to similar conclusions, demonstrating the presence of "platelet dust" in plasma. These two reports are probably the first ever to describe what we now call microparticles. To understand what O'BRIEN [5] and Wolf [6] meant by the expressions "platelet-like activity" and "platelet dust", we must refer to the classical view of the coagulation cascade, a simplified version of which, limited to the so-called extrinsic pathway, is depicted in figure 2. The coagulation cascade comprises a series of enzymatic reactions, each activating a proenzyme that becomes able to activate the next. Eventually, the enzyme thrombin (also known as FIIa) cleaves fibrinogen, generating fibrin that represents the insoluble matrix of the clot. It has been known for decades that several steps of the cascade require negatively charged phospholipids for the reaction to take place with the appropriate kinetics. Activated platelets have long been considered the main source of such membranes due to their small size, and therefore high surface/volume ratio, and to the fact

FIGURE 2 Simplified scheme of the "extrinsic" pathway of blood coagulation. F: factor.

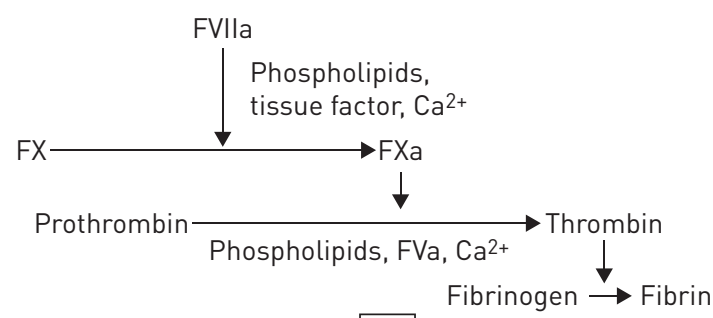

Phosphatidylcholine 
that upon activation platelets externalise the negatively charged phospholipid, phosphatidylserine [7]. What O'Brien [5] and Wolf [6] described was the presence, in both serum and plasma, of structures able to support the aforementioned reactions; these fluids, by definition cell-free environments, must therefore contain negatively charged phospholipids that are not cell-associated. These considerations led to the concept that negatively charged membrane fragments are released from platelets.

\section{Vascular microparticles beyond platelet dust}

Besides platelets, other vascular cells shed microparticles, including endothelial cells [8], leukocytes [9] and erythrocytes [10]. As previously mentioned, these microparticles have a procoagulant potential due to the presence of phosphatidylserine on their surface [11]. However, the presence of tissue factor (TF), the initiator of the so-called extrinsic coagulation pathway (figure 2), on the surface of some microparticles adds to their procoagulant potential [11]. Accordingly, numerous studies have demonstrated a role for microparticles of different cell origin in blood coagulation and thrombosis, both in vitro and in vivo [12-16]. However, besides $\mathrm{TF}$, microparticles have the potential to carry on their surface, and to enclose within their cytoplasm, other molecules derived from the parental cells, including proteins, DNA, RNA and microRNAs. Over the years, a picture has emerged that indicates that microparticles represent functional units with the potential of representing a disseminated storage pool of bioactive effectors, neither soluble nor cell-associated, that participate both in the maintenance of homeostasis and in the pathogenesis of disease [17].

\section{Mechanisms involved in microparticle formation}

The mechanisms involved in microparticle formation have only partially been elucidated; however, two distinct, well-characterised pathways have been identified, namely cell activation and apoptosis [18]. Microparticle shedding caused by activation starts within minutes after the addition of the relevant agonist [19] and is characterised by an increase in cytosolic calcium concentration [20]. In contrast, in apoptosis-dependent microparticle formation, dynamic membrane blebbing follows cell contraction and DNA fragmentation, and its time-course is usually measured in hours [21]. Several studies have demonstrated that inhibition of the increase in cytosolic calcium prevents microparticle formation. CAMPBELl et al. [22] demonstrated that the release of microparticles by S49 mouse lymphoma cells induced by ionomycin was inhibited by EGTA, a calcium chelator. We have shown that EGTA inhibits peroxisome proliferator-activated receptor (PPAR)- $\gamma$-induced release of microparticles by human monocytes/ macrophages (HMMs) [23]. In a similar model, using cigarette smoke extract as a stimulus for microparticle release, we also inhibited microparticle generation with the L-type calcium channel blocker verapamil [24]. Finally, the demonstration that calmodulin inhibition prevents cigarette smoke extract-induced microparticle generation indicates a role for this calcium-binding messenger protein [24].

Different key regulators of the cytoskeleton have been identified in microparticle generation. BuRGER et al. [8] demonstrated that Rho kinases are involved in endothelial cell-derived microparticle (EMP) formation, since Rho kinase inhibition blocked angiotensin II-induced release of microparticles. Furthermore, increased Rho kinase activity causes the release of circulating EMPs [25]. In addition, the small GTPase RhoA triggers a specific signalling pathway essential for microparticle biogenesis in various human cancer cells [26].

Other groups have demonstrated the involvement of mitogen-activated protein kinases (MAPKs) in microparticle generation. For example, activating autoantibodies to the angiotensin II receptor type 1 obtained from hypertensive patients promote EMP generation through the activation of p38 MAPK signalling, and p38 inhibitors effectively block this phenomenon [27]. Two groups have independently shown that the p38 pathway is involved in EMP generation upon stimulation with indoxyl sulphate [28] and tumour necrosis factor (TNF)- $\alpha$ [29]. In macrophage-derived microparticles obtained after stimulation with tobacco smoke extract, the production of microparticles relies on a series of dynamic, regulated steps that include activation of the c-Jun N-terminal kinase (JNK) and p38 MAPKs [30]. In the same model, other researchers have demonstrated that tobacco smoke extract exposure causes activation of JNK, p38 and extracellular signal-regulated kinase (ERK) MAPKs, as well as apoptosis, a major mechanism for microparticle generation, and that only the inhibition of ERK, but not p38 or JNK, significantly blunted tobacco smoke extract-induced microparticle generation [31]. In addition, we have demonstrated the involvement of the ERK pathway in the generation of microparticles by human monocytes stimulated with PPAR- $\gamma$ agonists [23].

Recently, various studies have investigated the involvement of nuclear factor (NF)- $\kappa \mathrm{B}$ in microparticle generation. Johnson et al. [32] studied the mechanisms involved in the release of neutrophil-derived microparticles (NMPs) after TNF- $\alpha$ stimulation, proving that the inhibition of NF- $\kappa$ B abrogates NMP generation induced by activation of both TNF- $\alpha$ receptors, TNF1 and TNF2. The same results were obtained in different cells by LeE et al. [33], who demonstrated the role of NF- $\kappa \mathrm{B}$ in the release of human umbilical vein EMPs. 


\section{Potential role of microparticles in airway inflammation}

The recruitment of blood-borne leukocytes into the airways represents a critical step in inflammatory reactions and requires the orchestrated action of cytokines, chemokines and cell-cell adhesion molecules [34]. Microparticles contribute to the production of various pro-inflammatory mediators by lung cells, thus becoming potential key factors in the airway inflammatory process. We have demonstrated that HMMs stimulated with the calcium ionophore A23187 and histamine generate microparticles with a pro-inflammatory potential for human lung cells. These microparticles, incubated with bronchial and alveolar epithelial cells, upregulate interleukin (IL)-8, monocyte chemotactic protein-1 and intercellular adhesion molecule-1 synthesis [35]. We investigated the mechanisms leading to increased transcription of pro-inflammatory mediators induced by HMM-derived microparticles. First, we demonstrated that HMM-derived microparticles cause the translocation of $\mathrm{NF}-\kappa \mathrm{B}$ into the nucleus [36]. We then showed that PPAR- $\gamma$ agonists, such as rosiglitazone and 15 -deoxy- $\Delta^{12,14}$-prostaglandin- $\mathrm{J}_{2}$, inhibit the phenomenon [36], in keeping with the postulated anti-inflammatory role of these molecules that have in fact been proposed as potential therapeutic targets in lung diseases [37]. Microparticles with a similar pro-inflammatory potential on lung epithelial cells are also generated by HMMs exposed to cigarette smoke extract [24]. In addition, FogLi et al. [38] demonstrated that HMM-derived microparticles induce a pro-inflammatory phenotype in human bronchial smooth muscle cells and that montelukast, a cysteinyl leukotriene receptor antagonist, reverses the phenomenon. In particular, montelukast prevented nuclear translocation of NF- $\mathrm{KB}$, blocked IL-8 and prostaglandin $\mathrm{E}_{2}$ release and restored salbutamol response in microparticle-stimulated human bronchial smooth muscle cells [38]. Finally, another group has demonstrated that human lymphoblastoma T-cell-derived microparticles induce the production of inflammatory cytokines, including TNF- $\alpha$, IL- 6 and IL-8, in a dose- and time-dependent manner by human bronchial cells [39].

Complementary experimental approaches involving animal models have provided many insights that contribute to the understanding of the relationships between airway inflammation and microparticle generation. Porro et al. [40] demonstrated that microparticles isolated from the sputum of cystic fibrosis patients are pro-inflammatory when injected into the murine lung, determine a strong neutrophilic infiltrate in the parenchyma and at the perivascular/peribronchial level, and that, among the possible mediators involved, lipopolysaccharide has a putative role in this phenomenon. In addition, exogenous EMPs injected into C57BL/6 mice caused a significant rise in pulmonary capillary permeability both as a primary and secondary injury [41].

\section{Microparticles in human lung diseases}

Microparticles have been investigated in several human lung diseases as possible pathogenic elements, prognostic markers and therapeutic targets. The data reported in the following sections are summarised in table 1.

\section{Chronic obstructive pulmonary disease}

Chronic obstructive pulmonary disease (COPD) is associated with several relevant comorbidities, possibly linked together through systemic inflammation [65-67]; among such comorbidities, cardiovascular diseases are probably the most significant, especially in prognostic terms [68-73]. It is of note that the presence of cardiovascular abnormalities has been demonstrated in a significant proportion of patients affected by COPD, even in the absence of clinically evident cardiac disease [74-76]. These close associations might be mediated by both endothelial dysfunction and activation, indicating an active pathogenic role played by endothelial cells $[77,78]$ as well as by lung vasculature damage and apoptosis. The latter is particularly relevant in the pathogenesis of emphysema and attributed, at least in part, to reduced function and/or levels of vascular endothelial growth factor [79-81].

Based on these theoretical considerations, the potential role of EMPs as possible pathogenic effectors and/ or prognostic markers in emphysema and COPD has been recently investigated. In a study by GORDON et al. [42], plasma EMPs were measured in 92 subjects, divided into healthy nonsmokers, healthy smokers with normal pulmonary function tests and normal diffusing capacity of the lung for carbon monoxide (DLCO), and "early emphysema" patients with normal pulmonary function tests but reduced DLCO. Smokers with reduced DLCO had significantly higher EMP levels than the other two groups, suggesting that EMPs could represent a potential marker of early emphysema. As previously mentioned, microparticles may originate either from cell activation or apoptosis. Using validated surface markers, Gordon et al. [42] were able to differentiate activated (high $\mathrm{CD}_{2} \mathrm{E}^{+}$to $\mathrm{CD} 31^{+}$ratio) from apoptotic (low $\mathrm{CD}_{2} \mathrm{E}^{+}$to $\mathrm{CD} 1^{+}$ratio) EMPs and to demonstrate a high percentage of apoptotic EMPs in early emphysema. Furthermore, the presence of angiotensin-converting enzyme on the surface of most EMPs indicates their pulmonary capillary origin. Taken together, these observations are consistent with the previously mentioned hypothesis that emphysema develops from a vascular bed injury in the lung and that EMPs represent a suitable tool to investigate these phenomena. 
TABLE 1 Summary of the known roles of microparticles (MPs) in human lung diseases

\begin{tabular}{|c|c|c|}
\hline Disease and MP type & Medium & Significance or hypothesised role \\
\hline \multicolumn{3}{|l|}{ COPD } \\
\hline \multirow[t]{3}{*}{ EMPs } & Plasma & $\begin{array}{l}\text { Markers of emphysema: increased apoptotic }\left(\mathrm{CD} 31^{+}\right) \mathrm{MPs} \text { in early } \\
\text { emphysema and mild COPD }\end{array}$ \\
\hline & & $\begin{array}{l}\text { Prognostic markers for exacerbation susceptibility: increased apoptotic } \\
\left.\left(\mathrm{CD} 31^{+}\right) \text {and activated (CD62 } \mathrm{E}^{+}\right) \mathrm{MPs} \text { during exacerbations; increased } \\
\text { activated MPs in stable COPD at higher risk for future exacerbations }\end{array}$ \\
\hline & & $\begin{array}{l}\text { Prognostic markers for functional decline over time: correlation of } \\
\text { number of activated }\left(\mathrm{CD}_{2} \mathrm{E}^{+}\right) \mathrm{MPs} \text { and FEV1 decline }\end{array}$ \\
\hline
\end{tabular}

$\begin{array}{ll}\begin{array}{ll}\text { Asthma } \\ \text { PMPs }\end{array} & \text { Plasma } \\ \text { TF-bearing MPs } & \text { BALF } \\ \text { Diffuse parenchymal lung disease } & \\ \text { PMPs and MMPs } & \text { Plasma } \\ \text { TF-bearing MPs } & \text { BALF } \\ \text { ARDS/ALI } & \\ \text { Total MPs } & \text { BALF } \\ \text { LMPs } & \text { Plasma } \\ \text { Pulmonary embolism and VTE } & \text { Plasma } \\ \text { EMPs } & \text { Plasma } \\ \text { TF-bearing MPs } & \text { Plasma } \\ & \\ \text { Lung cancer } & \text { Plasma } \\ \text { PMPs and MMPs } & \text { Plasma } \\ \text { Total MPs, PMPs and EMPs } & \\ \text { Pulmonary hypertension } & \text { Plasma } \\ \text { TF-bearing MPs } & \text { Plasma } \\ \text { EMPs } & \end{array}$

Role in organising bronchial inflammation

Promoting hypercoagulability during asthma exacerbations

Markers of lung involvement in systemic sclerosis

Markers of disease severity; promoting myofibroblast differentiation and activity

Local activation of the coagulation cascade; possible target for treatment Prognostic markers: increased in survivors

Promoting clot formation? (inconclusive data)

Promoting clotting in cancer patients

$[15,52]$

[53-56]

Possible markers for therapeutic decisions (i.e. thromboprophylaxis)

Promoting hypercoagulability and tumour angiogenesis (CD $31^{+}$CD $42 b^{-}$annexinV ${ }^{-}$) in survivors

Prothrombotic role in advanced $\mathrm{PAH}$

[62]

Markers of endothelial damage

[62]

Markers of different pathogenic patterns

[63]

Prognostic markers

COPD: chronic obstructive pulmonary disease; EMPs: endothelial cell-derived microparticles; FEV1: forced expiratory volume in 1 s; PMPs: platelet-derived microparticles; TF: tissue factor; BALF: bronchoalveolar lavage fluid; MMPs: monocyte-derived microparticles; ARDS: acute respiratory distress syndrome; ALI: acute lung injury; LMPs: leukocyte-derived microparticles; VTE: venous thromboembolism; PAH: pulmonary arterial hypertension.

Thomashow et al. [43] analysed EMPs from 104 COPD patients and 76 controls, and found a significant increase in $\mathrm{CD}_{31} 1^{+}$EMP levels (identified as apoptotic microparticles) in mild COPD, whereas CD62 $\mathrm{E}^{+}$ EMPs (identified as activated microparticles) were higher in severe COPD. Additionally, apoptotic EMP levels were positively correlated with the percentage of emphysema, measured by computed tomography scan, and negatively correlated with pulmonary microvasculature perfusion, as assessed by magnetic resonance imaging. Again, these observations suggest that pulmonary endothelial apoptosis could represent an early step in emphysema development.

TAKAHASH et al. [44] found a significant increase in both apoptotic and activated (CD31 ${ }^{+}$and $\mathrm{CD}^{+} \mathrm{E}^{+}$, respectively) EMPs during exacerbations compared with stable COPD. Although expression of angiotensin-converting enzyme was not assessed in this study, since the large majority of EMPs did not express von Willebrand factor (a marker of systemic vasculature not expressed by pulmonary capillaries), it was concluded that during an exacerbation pulmonary capillaries were mostly affected, both in terms of apoptosis and activation/inflammation. Furthermore, the study showed that elevated baseline levels of $\mathrm{CD} 2 \mathrm{E}^{+}$EMPs, suggestive of a sustained endothelial activation, even in stable COPD patients could predict a susceptibility to exacerbations, thus representing a possible prognostic marker. In a later study by the same investigators, a significant correlation between baseline CD62 $\mathrm{E}^{+}$EMP levels and annual change in forced expiratory volume in $1 \mathrm{~s}$ (FEV1) was found: higher EMP levels predicted a more relevant decline in FEV1, suggesting that a persistent endothelial activation and inflammation could play a role in the functional decline of COPD patients [45].

Thus, analysis of pulmonary endothelial cell activation and apoptosis through EMP evaluation is proving instrumental in generating data that lend further support to the hypothesis that COPD is a disease with a 
FIGURE 3 Proposed interactions among the environment, lungs, endothelium, bone marrow and adipose tissue in the pathogenesis of chronic obstructive pulmonary disease and its comorbidities. Reproduced and modified from [82] with permission.

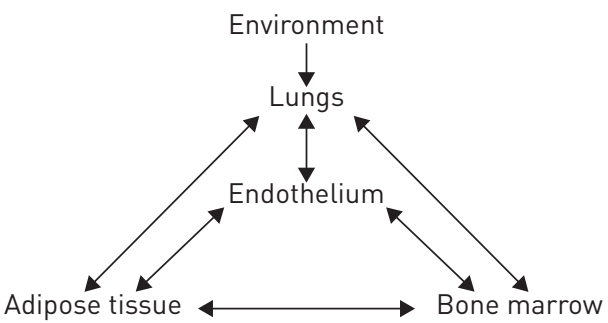

significant endothelial component, which could link lung disease and its systemic (and especially cardiovascular) comorbidities [78]. Agustí et al. [82] recently proposed a general model whereby the lungs, endothelium, bone marrow and adipose tissue form a network, in which the lung represents an external sensor, the endothelium an internal sensor and the bone marrow and adipose tissue the responsive elements. The model, summarised in figure 3, is largely speculative and AGustí et al. [82] acknowledged that much experimental work is needed in order to thoroughly identify the links between the lungs and the other components. Microparticles may represent one such link. Notably, we have reported that both leptin, an adipokine secreted by adipose tissue [83], and airborne pollutants [84] cause microparticle generation in macrophages and endothelial cells.

\section{Asthma}

Asthma is usually characterised by different patterns of airway inflammation, with a complex network of cellular and molecular mediators [85-87]. In contrast to COPD, very little is known about the possible role of microparticles in asthma and the scarce available data are preliminary, so that further research in the field, through properly designed studies, is needed. In a recent study conducted on 35 subjects (20 individuals with asthma and 15 healthy controls), asthmatic patients showed significantly higher baseline levels of platelet-derived microparticles (PMPs) than controls [46]. It is worth noting that all asthmatic patients were taking inhaled steroids. This finding could mark a potential pathogenic role for PMPs in airways inflammation, especially in organising cellular (in particular leukocyte) trafficking between blood and bronchi.

The interplay between inflammation and blood coagulation is well recognised; accordingly, an increased risk of pulmonary embolism in asthmatics, with an association with exacerbations, has been described [88, 89]. MAJOor et al. [47] investigated the role of viral infections in the haemostatic balance in asthmatic subjects. They showed an increase in TF-bearing microparticles in the bronchoalveolar lavage fluid (BALF) of asthmatics after experimental rhinovirus infection. They hypothesised that TF-bearing microparticles could play a pathogenic role in the local activation of coagulation during asthma infectious exacerbations, although the population sample was too small (14 individuals with asthma and 14 healthy controls) to allow for definite conclusions.

\section{Diffuse parenchymal lung diseases}

Diffuse parenchymal lung diseases represent a very inhomogeneous group of pulmonary diseases [90], with several different aetiologies and pathogenic patterns, most of them not clearly understood or, in fact, even almost completely unknown. Moreover, many of these conditions are quite rare. Very few studies have addressed the potential role of microparticles in diffuse parenchymal lung diseases. NomURA et al. [48] showed, in a small sample of 42 patients with progressive systemic sclerosis, that levels of PMPs and monocyte-derived microparticles (MMPs) were increased compared with 30 healthy controls. More interestingly, significantly higher levels of PMPs and MMPs were found in patients with pulmonary involvement (i.e. with interstitial lung disease), compared with patients without lung disease. Such elevated levels of microparticles might therefore represent a possible marker of more advanced disease and a putative marker to stratify patients when choosing a treatment strategy, such as anti-platelet drugs.

A link between blood coagulation and pulmonary fibrosis is well recognised [91]. Based on the role of microparticles in blood coagulation, we investigated their presence in diffuse parenchymal lung disease patients [49]. We showed an increased number of microparticles in the BALF of 19 patients with interstitial lung diseases compared with 11 control subjects. Furthermore, the microparticle-bound TF (MP-TF) procoagulant activity was significantly higher in patients than in controls. When patients were further divided into idiopathic pulmonary fibrosis (IPF) and non-IPF groups, the MP-TF procoagulant activity was significantly higher in the former group. Additionally, a statistically significant negative correlation was found between TF-bearing microparticles and both forced vital capacity and DLCO in IPF patients [49]. In the same study, in vitro assays showed that an oxidative stimulus, namely $\mathrm{H}_{2} \mathrm{O}_{2}$, increased the production of procoagulant microparticles by alveolar epithelial cells in culture [49]. Local synthesis of 
coagulation factor $\mathrm{X}$ and its activation to factor $\mathrm{Xa}$ in the lung has been implicated in the pathogenesis of IPF. Factor $\mathrm{Xa}$ is a protease that, besides its well-known role in cleaving prothrombin to generate thrombin, is also capable of cleaving the protease-activated receptor (PAR)-1 on fibroblasts, signalling their differentiation into myofibroblasts and therefore potentially contributing to the fibrotic process [92]. Increased local synthesis of factor X and local expression of factor Xa has been demonstrated in IPF lungs [92]. Our data are consistent with the existence of a pathway whereby an oxidative stress induces the generation of procoagulant, TF-bearing microparticles that activate locally synthesised factor $\mathrm{X}$ to $\mathrm{Xa}$, thus inducing a PAR-1-mediated activation of fibroblasts and a profibrotic response.

\section{Acute respiratory distress syndrome}

In acute respiratory distress syndrome (ARDS) a burst of local inflammation is present, associated with an activation of the coagulation cascade and a significant deposition of fibrin in the alveolar spaces. Based on previous evidence of the activation of the TF pathway in ARDS [93], BASTARACHE et al. [50] found a significantly higher concentration of microparticles in pulmonary oedema fluid from ARDS subjects than from patients with cardiogenic pulmonary oedema. Moreover, microparticles from ARDS patients had a higher procoagulant activity compared with those from patients with cardiogenic oedema. This procoagulant activity was mainly due to the presence of increased concentration of TF in the fluid, which strongly correlated with microparticle levels, indicating the presence of TF-bearing microparticles. As suggested by subsequent in vitro analysis, the alveolar epithelium probably represented the main source of microparticles in ARDS. Furthermore, the study showed a trend towards lower microparticle concentrations in ARDS patients who survived compared with those who died of the disease. BASTARACHE et al. [50] speculated that microparticles could play a key role in the activation of blood coagulation and in the deposition of fibrin in the alveolar spaces that characterises ARDS, and that they might represent a potential target for future treatment of ARDS.

Apparently discordant results emerged from another study that analysed plasma and BALF levels of microparticles of different cellular origin (leukocytes, neutrophils, endothelium and platelets) in 52 patients with ARDS and 22 controls [51]. Higher plasmatic levels of leukocyte-derived microparticles (LMPs) were associated with a better prognosis in the ARDS group, a result apparently at odds with that of BASTARACHE et al. [50]; in fact, these results only suggest that circulating LMPs, as opposed to PMPs and MMPs, might be protective, possibly through their positive effects on vascular tone, as speculated in previous works on sepsis and septic shock [94].

Finally, in a murine model, the injection of EMPs into mice induced a significant release of the pro-inflammatory cytokines TNF- $\alpha$ and IL-1 $\beta$, with a subsequent recruitment of neutrophils [95]. These effects were further increased by the concomitant or sequential administration of bacterial lipopolysaccharide, indicating that EMPs might represent a signal that primes the lung for the following inflammatory response to an external injury. Again, from this point of view, EMPs could represent a potential therapeutic target for ARDS. In conclusion, these studies have mainly hypothesised a role for microparticles (especially for EMPs) in the pathogenesis of ARDS: in particular, EMPs could represent a link between alveolar inflammation and coagulation (two key steps in the pathobiology of ARDS), and perhaps even a target for future treatment.

\section{Pulmonary embolism}

The potential role of procoagulant microparticles in venous thromboembolism (VTE) has been extensively investigated. A first series of studies measured the level of circulating microparticles, irrespective of their MP-TF procoagulant activity. CHIRINOs et al. [15] found an increase in plasma levels of EMPs and of EMP-monocyte conjugates in 25 patients with VTE compared with 25 healthy controls, suggesting that during VTE episodes EMPs and their interactions with leukocytes could play a role in clot formation. From this point of view, EMPs might represent a potential therapeutic target for preventing and treating thromboembolic events.

In contrast, in another study, EMP and PMP levels were similar between patients with acute pulmonary embolism and control subjects [52]. A difference in circulating EMP and PMP levels was found when the pulmonary embolism group was compared with the control subjects without cardiovascular risk factors, but disappeared when controls with cardiovascular risk factors were included [52], suggesting that circulating microparticles simply represent markers of such cardiovascular risk factors.

A more recent study showed higher levels of total (i.e. irrespective of their cellular origin) phosphatidylserine-positive circulating microparticles in 186 patients with VTE compared with 418 healthy controls [96]. Furthermore, there was a positive correlation between microparticle levels and risk of a first VTE episode. Since the composition of microparticles gave biological plausibility to the hypothesis of their prothrombotic properties, the study suggested a pathogenic role for circulating microparticles in VTE events. 
While these reports did not investigate the possible functional role of MP-TF in clot formation, other studies have mainly focused on in vitro tests for MP-TF procoagulant activity of circulating microparticles, to better elucidate the pathophysiological relationship between microparticles and VTE. In a retrospective study by GARCIA RODRIGUEz et al. [97], the procoagulant activity of TF-bearing microparticles (MP-TF activity) was higher in patients with suspected pulmonary embolism than in controls; however, there were no significant differences between MP-TF activity in patients with confirmed pulmonary embolism and patients without the disease. Since an exploratory analysis in the group with suspected pulmonary embolism revealed higher levels of MP-TF activity in patients affected by cancer, GARCIA RodRIGUEz et al. [97] concluded that MP-TF activity was not part of the VTE event, but rather reflected the presence of cancer. The prospective study by THALER et al. [98], conducted on patients with deep vein thrombosis (with or without pulmonary embolism), came to similar conclusions and did not support the hypothesis that TF-bearing microparticles play a clear role in the pathogenesis of thromboembolic events. In conclusion, functional studies on MP-TF activity failed to definitively confirm the hypothesis that emerged from previous studies that investigated the levels of circulating microparticles.

The association of VTE with cancer is well established [99] and the role of microparticles in this setting has also been investigated. In a study by TesseLAar et al. [53], in patients with VTE and metastatic cancer, MP-TF activity was higher than in patients with cancer but no VTE, in healthy subjects or in patients with idiopathic VTE. These findings suggest the possibility that tumour-derived microparticles might initiate the clotting cascade in vivo in cancer patients, thus playing a pathogenic role. Similar results derive from other studies that confirm that MP-TF activity is associated with cancer, with an "overactivity" in the presence of a VTE event [54-56]. These findings support the hypothesis that these microparticles are produced by tumour cells, play a pathogenic role in the development of VTE and may represent a potential biomarker for critical clinical challenges such as, for example, the choice of the ideal prophylactic treatment in oncology patients. ZWICKER et al. [57], in a phase II randomised trial, divided a cohort of 66 patients with a nonresectable cancer, according to the levels of MP-TF activity (low and high levels); they further split the high-level group into two arms, according to the administration of a prophylactic dose of enoxaparin. Patients with high levels of MP-TF activity treated with enoxaparin showed a significantly lower cumulative incidence of VTE at 2 months compared with those not treated and with high levels of MP-TF activity, while the incidence was similar to the low-level group, suggesting that levels of MP-TF activity could be used as a potential biomarker to stratify the risk of VTE and drive the choice of a prophylactic anticoagulation strategy in cancer patients.

\section{Lung cancer}

Lung cancer represents the main cause of cancer death worldwide [100]. In order to reach an earlier diagnosis and to better select patients for individual treatments, several biomarkers have been studied, including microparticles, but with inconsistent results regarding the possible biological roles of microparticles in lung cancer. In an early study by KANAZAWA et al. [58], PMP and MMP levels were higher in 64 patients with lung cancer (both small and nonsmall cell lung cancer) compared with 30 controls. Additionally, in cancer patients, platelet activation markers were also higher than in controls, suggesting a potential biological relationship between microparticles (both MMPs and PMPs) and vascular complications (both hypercoagulability and tumour angiogenesis) in these subjects.

Other studies have confirmed the higher levels of different types of microparticles in patients with lung cancer compared with healthy controls. These have mostly focused on the possible prognostic value of microparticles in lung cancer but the results were discordant. FLEITAS et al. [59] found higher levels of total circulating microparticles in 60 patients with nonsmall cell lung cancer compared with 60 controls, but the most relevant finding was that, in cancer patients, higher baseline levels of microparticles were associated with a better progression-free and overall survival. Discordant results emerged from another study conducted in 107 end-stage nonsmall cell lung cancer patients, divided into 1-year survivors and nonsurvivors, measuring PMPs and EMPs [60]. While there was no difference in PMPs and "apoptotic" EMPs (defined as $\mathrm{CD} 31^{+} \mathrm{CD}_{4} 2 \mathrm{~b}^{-}$annexinV ${ }^{+}$microparticles) between the two groups, "activated" EMP levels (defined as $\mathrm{CD} 1^{+} \mathrm{CD} 42 \mathrm{~b}^{-}$annexinV $\mathrm{V}^{-}$microparticles) were significantly higher in nonsurvivors [60]. After a multivariate regression analysis, these higher levels were an independent predictor of 1-year mortality. This result was clearly in contrast with the study by Fleitas et al. [59]. Moreover, in a previous study on the topic by the same investigators [61], both PMP and EMP levels were higher in 130 lung cancer patients compared with 30 healthy controls, but they were not associated with the presence of metastasis or with cancer stage (early versus advanced). All these data underline the need for further studies in this field, in order to better elucidate the possible prognostic and therapeutic role of microparticles in lung cancer.

\section{Pulmonary arterial hypertension}

Pulmonary arterial hypertension (PAH) is a devastating disease, still characterised by a poor prognosis despite treatment [101], with several recognised pathogenic mechanisms, including increased coagulability 
and thrombosis, and endothelial dysfunction [102-106]. Microparticles have been investigated in animal models of PAH [107-109]. The results support the hypothesis that microparticles might act directly on endothelial function by altering vascular balance, through a reduction of nitric oxide production, an increase in oxidative stress and as a circulating source of vasoconstrictor agents such as thromboxane $\mathrm{A}_{2}$.

In human pulmonary hypertension, microparticles could represent both a pathogenic element and a prognostic marker. A pathogenic role for microparticles in humans has been proposed in a study on 20 patients affected by PAH [62]. These subjects showed higher levels of endoglin ${ }^{+}$EMPs compared with 23 controls. Moreover, patients had higher MP-TF activity than controls, with the highest values in more severe hypertensive subjects (World Health Organization (WHO) functional classes III and IV and 6-min walking distance $<380 \mathrm{~m}$ ). These data supported a theoretical relationship between endothelial damage and $\mathrm{PAH}$ severity. Furthermore, the highest levels of TF-bearing microparticle activity in the most severe subgroup of patients might underline the possible prothrombotic contribution of microparticles in the advanced stage of disease. Finally, since endoglin is an accessory receptor for transforming growth factor- $\beta$, involved in cell proliferation and in neoangiogenesis, EMPs could participate in the pathogenesis of plexiform lesions and vascular remodelling.

Another study supported a role for microparticles in the pathogenesis of PAH [110]. In this study, 10 patients with idiopathic PAH showed a significant increase of CD39 nucleotidase expression and function on microparticle membranes compared with 10 controls. Since CD39 has an ATP nucleotidase activity, and since ATP has an endothelium-dependent vasodilatory effect on pulmonary arteries, the CD39 increased activity could contribute to further raise pulmonary vascular resistance by reducing ATP endovascular concentration. Conversely, CD39 activity ultimately leads to a reduction in AMP concentration, and since AMP is a potent stimulator for platelet aggregation and thrombosis, the increased CD39 levels could also represent a compensatory mechanism in order to limit the pathogenic process.

Microparticles could also represent a marker of severity in pulmonary hypertension. AmABILE et al. [63] found increased levels of EMPs in an inhomogeneous sample of 24 patients with pre-capillary pulmonary hypertension, compared with 20 healthy controls. Although no relationships existed between EMPs and disease severity as assessed by WHO functional classes or 6-min walking test, there were significant positive correlations between haemodynamic parameters of disease severity and both CD $144^{+}$and CD31 $1^{+}$EMP levels, and between $\mathrm{CD}_{2} \mathrm{E}^{+}$EMP levels and systemic inflammation (evaluated with high-sensitivity C-reactive protein). Taken together, these results may indicate that different types of EMP can represent markers of different pathogenic pathways in pulmonary hypertension (with CD $144^{+}$and CD31 ${ }^{+}$EMP levels as predictors of disease severity and $\mathrm{CD}_{2} \mathrm{E}^{+} \mathrm{EMP}$ levels as markers of vascular inflammation), but also that EMPs could represent potential markers to predict prognosis and to perhaps monitor response to pharmacological treatments. In order to better elucidate these latter aspects, the same investigators analysed the same cohort in a prospective study, with a 1-year follow-up [64], and found significantly higher $\mathrm{CD}_{62} \mathrm{E}^{+} \mathrm{EMP}$ levels in pulmonary hypertension patients who developed an adverse outcome (death or worsening right heart failure) than in those with stable disease. Additionally, Amabile et al. [64] identified a cut-off value for $\mathrm{CD}_{62} \mathrm{E}^{+} \mathrm{EMP}$ levels to independently predict a worse prognosis. These data suggest that $\mathrm{CD} 2 \mathrm{E}^{+} \mathrm{EMP}$ levels could be used as a prognostic marker to better stratify pulmonary hypertension patients before starting a specific treatment. Other studies demonstrated higher levels of circulating microparticles in patients with pulmonary hypertension compared with healthy controls, but they did not assess the potential utility of microparticles as prognostic markers in the clinical setting $[111,112]$.

\section{Conclusive remarks and future directions}

Microparticles are rapidly gaining consideration both as biomarkers and as potential targets for therapeutic interventions. In the field of respiratory medicine, microparticles have, for example, the potential to help identify COPD phenotypes and stratify disease severity, to improve risk stratification for the development of VTE to better define prophylactic strategies, and to allow a better prognostic characterisation of ARDS patients. Furthermore, as a pathogenic role for microparticles is clearly emerging, the precise identification of the mechanisms involved in their formation is likely to prove valuable in identifying much needed novel therapeutic targets.

\section{References}

1 van der Pol E, Böing AN, Harrison P, et al. Classification, functions, and clinical relevance of extracellular vesicles. Pharmacol Rev 2012; 64: 676-705.

2 György B, Szabó TG, Pásztói M, et al. Membrane vesicles, current state-of-the-art: emerging role of extracellular vesicles. Cell Mol Life Sci 2011; 68: 2667-2688.

Burger D, Schock S, Thompson CS, et al. Microparticles: biomarkers and beyond. Clin Sci 2013; 124: 423-441. Geddings JE, Mackman N. New players in haemostasis and thrombosis. Thromb Haemost 2014; 111: 570-574. O'Brien JR. The platelet-like activity of serum. Br J Haematol 1955; 1: 223-228.

Wolf P. The nature and significance of platelet products in human plasma. Br J Haematol 1967; 13: 269-288. 
Furie B, Furie BC. The molecular basis of blood coagulation. Cell 1988; 53: 505-518.

Burger D, Montezano AC, Nishigaki N, et al. Endothelial microparticle formation by angiotensin II is mediated via Ang II receptor type I/NADPH oxidase/Rho kinase pathways targeted to lipid rafts. Arterioscler Thromb Vasc Biol 2011; 31: 1898-1907.

Wang JG, Aikawa E, Aikawa M. Leukocyte-derived microparticles as proinflammatory mediators in atherosclerosis. J Am Coll Cardiol 2013; 62: 1442-1445.

Koshiar RL, Somajo S, Norström E, et al. Erythrocyte-derived microparticles supporting activated protein C-mediated regulation of blood coagulation. PLoS One 2014; 9: e104200.

Celi A, Lorenzet R, Furie BC, et al. Microparticles and a P-selectin-mediated pathway of blood coagulation. Dis Markers 2004; 20: 347-352.

Falati S, Liu Q, Gross P, et al. Accumulation of tissue factor into developing thrombi in vivo is dependent upon microparticle P-selectin glycoprotein ligand 1 and platelet P-selectin. J Exp Med 2003; 197: 1585-1598.

Chou J, Mackman N, Merrill-Skoloff G, et al. Hematopoietic cell-derived microparticle tissue factor contributes to fibrin formation during thrombus propagation. Blood 2004; 104: 3190-3197.

Steppich B, Mattisek C, Sobczyk D, et al. Tissue factor pathway inhibitor on circulating microparticles in acute myocardial infarction. Thromb Haemost 2005; 93: 35-39.

Chirinos JA, Heresi GA, Velasquez $\mathrm{H}$, et al. Elevation of endothelial microparticles, platelets, and leukocyte activation in patients with venous thromboembolism. J Am Coll Cardiol 2005; 45: 1467-1471.

Hu SS, Zhang HG, Zhang QJ, et al. Small-size circulating endothelial microparticles in coronary artery disease. PLoS One 2014; 9: e104528.

Hugel B, Martínez MC, Kunzelmann C, et al. Membrane microparticles: two sides of the coin. Physiology 2005; 20: 22-27.

VanWijk MJ, VanBavel E, Sturk A, et al. Microparticles in cardiovascular diseases. Cardiovasc Res 2003; 59: 277-287. MacKenzie A, Wilson HL, Kiss-Toth E, et al. Rapid secretion of interleukin-1 $\beta$ by microvesicle shedding. Immunity 2001; 15: 825-835.

Wiedmer T, Sims PJ. Participation of protein kinases in complement C5b-9-induced shedding of platelet plasma membrane vesicles. Blood 1991; 78: 2880-2886.

Aupeix K, Hugel B, Martin T, et al. The significance of shed membrane particles during programmed cell death in vitro, and in vivo, in HIV-1 infection. J Clin Invest 1997; 99: 1546-1554.

Campbell LE, Nelson J, Gibbons E, et al. Membrane properties involved in calcium-stimulated microparticle release from the plasma membranes of S49 lymphoma cells. Scientific World Journal 2014; 2014: 537192.

Neri T, Cordazzo C, Carmazzi Y, et al. Effects of peroxisome proliferator-activated receptor- $\gamma$ agonists on the generation of microparticles by monocytes/macrophages. Cardiovasc Res 2012; 94: 537-544.

Cordazzo C, Petrini S, Neri T, et al. Rapid shedding of proinflammatory microparticles by human mononuclear cells exposed to cigarette smoke is dependent on $\mathrm{Ca}^{2+}$ mobilization. Inflamm Res 2014; 63: 539-547.

Gao C, Li R, Liu Y, et al. Rho-kinase-dependent F-actin rearrangement is involved in the release of endothelial microparticles during IFN- $\alpha$-induced endothelial cell apoptosis. J Trauma Acute Care Surg 2012; 73: 1152-1160.

Li B, Antonyak MA, Zhang J, et al. RhoA triggers a specific signaling pathway that generates transforming microvesicles in cancer cells. Oncogene 2012; 31: 4740-4749.

Yang S, Zhong Q, Qiu Z, et al. Angiotensin II receptor type 1 autoantibodies promote endothelial microparticles formation through activating p38 MAPK pathway. J Hypertens 2014; 32: 762-770.

Ryu JH, Kim SJ. Clopidogrel effectively suppresses endothelial microparticle generation induced by indoxyl sulfate via inhibition of the p38 mitogen-activated protein kinase pathway. Blood Purif 2011; 32: 186-194.

Curtis AM, Wilkinson PF, Gui M, et al. p38 mitogen-activated protein kinase targets the production of proinflammatory endothelial microparticles. J Thromb Haemost 2009; 7: 701-709.

Li CJ, Liu Y, Chen Y, et al. Novel proteolytic microvesicles released from human macrophages after exposure to tobacco smoke. Am J Pathol 2013; 182: 1552-1562.

$\mathrm{Li} \mathrm{M}$, Yu D, Williams KJ, et al. Tobacco smoke induces the generation of procoagulant microvesicles from human monocytes/macrophages. Arterioscler Thromb Vasc Biol 2010; 30: 1818-1824.

Johnson BL 3rd, Goetzman HS, Prakash PS, et al. Mechanisms underlying mouse TNF- $\alpha$ stimulated neutrophil derived microparticle generation. Biochem Biophys Res Commun 2013; 437: 591-596.

Lee SK, Yang SH, Kwon I, et al. Role of tumour necrosis factor receptor-1 and nuclear factor- $\kappa \mathrm{B}$ in production of TNF- $\alpha$-induced pro-inflammatory microparticles in endothelial cells. Thromb Haemost 2014; 112: 580-588.

Chung KF, Adcock IM. Multifaceted mechanisms in COPD: inflammation, immunity, and tissue repair and destruction. Eur Respir J 2008; 31: 1334-1356.

Cerri C, Chimenti D, Conti I, et al. Monocyte/macrophage-derived microparticles up-regulate inflammatory mediator synthesis by human airway epithelial cells. J Immunol 2006; 177: 1975-1980.

Neri T, Armani C, Pegoli A, et al. Role of NF- $\kappa B$ and PPAR- $\gamma$ in lung inflammation induced by monocyte-derived microparticles. Eur Respir J 2011; 37: 1494-1502.

Belvisi MG, Hele DJ. Peroxisome proliferator-activated receptors as novel targets in lung disease. Chest 2008; 134 : 152-157.

Fogli S, Stefanelli F, Neri T, et al. Montelukast prevents microparticle-induced inflammatory and functional alterations in human bronchial smooth muscle cells. Pharmacol Res 2013; 76: 149-156.

Qiu Q, Xiong W, Yang C, et al. Lymphocyte-derived microparticles induce bronchial epithelial cells' pro-inflammatory cytokine production and apoptosis. Mol Immunol 2013; 55: 220-230.

Porro C, Di Gioia S, Trotta T, et al. Pro-inflammatory effect of cystic fibrosis sputum microparticles in the murine lung. J Cyst Fibros 2013; 12: 721-728.

Densmore JC, Signorino PR, Ou J, et al. Endothelium-derived microparticles induce endothelial dysfunction and acute lung injury. Shock 2006; 26: 464-471.

Gordon C, Gudi K, Krause A, et al. Circulating endothelial microparticles as a measure of early lung destruction in cigarette smokers. Am J Respir Crit Care Med 2011; 184: 224-232.

Thomashow MA, Shimbo D, Parikh MA, et al. Endothelial microparticles in mild chronic obstructive pulmonary disease and emphysema. The Multi-Ethnic Study of Atherosclerosis Chronic Obstructive Pulmonary Disease study. Am J Respir Crit Care Med 2013; 188: 60-68. 
44

Takahashi T, Kobayashi S, Fujino N, et al. Increased circulating endothelial microparticles in COPD patients: a potential biomarker for COPD exacerbation susceptibility. Thorax 2012; 67: 1067-1074.

Takahashi T, Kobayashi S, Fujino N, et al. Annual FEV1 changes and numbers of circulating endothelial microparticles in patients with COPD: a prospective study. BMJ Open 2014; 4: e004571.

Duarte D, Taveira-Gomes T, Sokhatska $\mathrm{O}$, et al. Increased circulating platelet microparticles as a potential biomarker in asthma. Allergy 2013; 68: 1073-1075.

Majoor CJ, van de Pol MA, Kamphuisen PW, et al. Evaluation of coagulation activation after rhinovirus infection in patients with asthma and healthy control subjects: an observational study. Respir Res 2014; 15: 14.

Nomura S, Inami N, Ozaki Y, et al. Significance of microparticles in progressive systemic sclerosis with interstitial pneumonia. Platelets 2008; 19: 192-198.

Novelli F, Neri T, Tavanti L, et al. Procoagulant, tissue factor-bearing microparticles in bronchoalveolar lavage of interstitial lung disease patients: an observational study. PLoS One 2014; 9: e95013.

Bastarache JA, Fremont RD, Kropski JA, et al. Procoagulant alveolar microparticles in the lungs of patients with acute respiratory distress syndrome. Am J Physiol Lung Cell Mol Physiol 2009; 297: L1035-L1041.

Guervilly C, Lacroix R, Forel JM, et al. High levels of circulating leukocyte microparticles are associated with better outcome in acute respiratory distress syndrome. Crit Care 2011; 15: R31.

Bal L, Ederhy S, Di Angelantonio E, et al. Factors influencing the level of circulating procoagulant microparticles in acute pulmonary embolism. Arch Cardiovasc Dis 2010; 103: 394-403.

Tesselaar ME, Romijn FP, Van Der Linden IK, et al. Microparticle-associated tissue factor activity: a link between cancer and thrombosis? J Thromb Haemost 2007; 5: 520-527.

Zwicker JI, Liebman HA, Neuberg D, et al. Tumor-derived tissue factor-bearing microparticles are associated with venous thromboembolic events in malignancy. Clin Cancer Res 2009; 15: 6830-6840.

Manly DA, Wang J, Glover SL, et al. Increased microparticle tissue factor activity in cancer patients with venous thromboembolism. Thromb Res 2010; 125: 511-512.

Campello E, Spiezia L, Radu CM, et al. Endothelial, platelet, and tissue factor-bearing microparticles in cancer patients with and without venous thromboembolism. Thromb Res 2011; 127: 473-477.

Zwicker JI, Liebman HA, Bauer KA, et al. Prediction and prevention of thromboembolic events with enoxaparin in cancer patients with elevated tissue factor-bearing microparticles: a randomized-controlled phase II trial (the Microtec study). Br J Haematol 2013; 160: 530-537.

Kanazawa S, Nomura S, Kuwana M, et al. Monocyte-derived microparticles may be a sign of vascular complication in patients with lung cancer. Lung Cancer 2003; 39: 145-149.

Fleitas T, Martínez-Sales V, Vila V, et al. Circulating endothelial cells and microparticles as prognostic markers in advanced non-small cell lung cancer. PLoS One 2012; 7: e47365.

Wang CC, Tseng CC, Hsiao CC, et al. Circulating endothelial-derived activated microparticle: a useful biomarker for predicting one-year mortality in patients with advanced non-small cell lung cancer. Biomed Res Int 2014; 2014: 173401.

Tseng CC, Wang CC, Chang HC, et al. Levels of circulating microparticles in lung cancer patients and possible prognostic value. Dis Markers 2013; 35: 301-310.

Bakouboula B, Morel O, Faure A, et al. Procoagulant membrane microparticles correlate with the severity of pulmonary arterial hypertension. Am J Respir Crit Care Med 2008; 177: 536-543.

Amabile N, Heiss C, Real WM, et al. Circulating endothelial microparticle levels predict hemodynamic severity of pulmonary hypertension. Am J Respir Crit Care Med 2008; 177: 1268-1275.

Amabile N, Heiss C, Chang V, et al. Increased CD62 $\mathrm{e}^{+}$endothelial microparticle levels predict poor outcome in pulmonary hypertension patients. J Heart Lung Transplant 2009; 28: 1081-1086.

Walter RE, Wilk JB, Larson MG, et al. Systemic inflammation and COPD: the Framingham Heart Study. Chest 2008; 133: 19-25.

Gan WQ, Man SF, Senthilselvan A, et al. Association between chronic obstructive pulmonary disease and systemic inflammation: a systematic review and a meta-analysis. Thorax 2004; 59: 574-580.

Agustí A, Faner R. Systemic inflammation and comorbidities in chronic obstructive pulmonary disease. Proc Am Thorac Soc 2012; 9: 43-46.

Mannino DM, Thorn D, Swensen A, et al. Prevalence and outcomes of diabetes, hypertension and cardiovascular disease in COPD. Eur Respir J 2008; 32: 962-969.

Schünemann HJ, Dorn J, Grant BJ, et al. Pulmonary function is a long-term predictor of mortality in the general population: 29-year follow-up of the Buffalo Health Study. Chest 2000; 118: 656-664.

Agustí A, Calverley PM, Celli B, et al. Characterisation of COPD heterogeneity in the ECLIPSE cohort. Respir Res 2010; 11: 122.

McGarvey LP, John M, Anderson JA, et al. Ascertainment of cause-specific mortality in COPD: operations of the TORCH Clinical Endpoint Committee. Thorax 2007; 62: 411-415.

Divo M, Cote C, de Torres JP, et al. Comorbidities and risk of mortality in patients with chronic obstructive pulmonary disease. Am J Respir Crit Care Med 2012; 186: 155-161.

Agustí A, Edwards LD, Rennard SI, et al. Persistent systemic inflammation is associated with poor clinical outcomes in COPD: a novel phenotype. PLoS One 2012; 7: e37483.

Iwamoto H, Yokoyama A, Kitahara Y, et al. Airflow limitation in smokers is associated with subclinical atherosclerosis. Am J Respir Crit Care Med 2009; 179: 35-40.

Barr RG, Ahmed FS, Carr JJ, et al. Subclinical atherosclerosis, airflow obstruction and emphysema: the MESA Lung Study. Eur Respir J 2012; 39: 846-854.

6 McAllister DA, Maclay JD, Mills NL, et al. Arterial stiffness is independently associated with emphysema severity in patients with chronic obstructive pulmonary disease. Am J Respir Crit Care Med 2007; 176: 1208-1214.

Clarenbach CF, Senn O, Sievi NA, et al. Determinants of endothelial function in patients with COPD. Eur Respir J 2013; 42: 1194-1204.

Barberà JA. Chronic obstructive pulmonary disease: a disease of the endothelium? Am J Respir Crit Care Med 2013; 188: 5-7.

Kasahara Y, Tuder RM, Taraseviciene-Stewart L, et al. Inhibition of VEGF receptors causes lung cell apoptosis and emphysema. J Clin Invest 2000; 106: 1311-1319. 

growth factor and vascular endothelial growth factor receptor 2 in emphysema. Am J Respir Crit Care Med 2001; 163: 737-744

81 Kanazawa H, Asai K, Hirata K, et al. Possible effects of vascular endothelial growth factor in the pathogenesis of chronic obstructive pulmonary disease. Am J Med 2003; 114: 354-358.

82 Agustí A, Barberà JA, Wouters EF, et al. Lungs, bone marrow, and adipose tissue. A network approach to the pathobiology of chronic obstructive pulmonary disease. Am J Respir Crit Care Med 2013; 188: 1396-1406.

83 Petrini S, Neri T, Lombardi S, et al. Leptin induces the generation of procoagulant, tissue factor bearing microparticles by human peripheral blood mononuclear cells. Biochim Biophys Acta 2016; 1860: $1354-1361$.

84 Neri T, Pergoli L, Petrini S, et al. Particulate matter induces prothrombotic microparticle shedding by human mononuclear and endothelial cells. Toxicol In Vitro 2016; 32: 333-338.

85 Global Initiative for Asthma. Global Strategy for Asthma Management and Prevention. 2016 Report. Available from http://ginasthma.org

86 Saglani S, Lloyd CM. Novel concepts in airway inflammation and remodelling in asthma. Eur Respir J 2015; 46: 1796-1804.

87 Pelaia G, Vatrella A, Busceti MT, et al. Cellular mechanisms underlying eosinophilic and neutrophilic airway inflammation in asthma. Mediators Inflamm 2015; 2015: 879783.

88 Majoor CJ, Bel EH. Allergic burden and the risk of venous thromboembolism. Eur Respir J 2013; 42: 1158-1159.

89 Chung WS, Lin CL, Ho FM, et al. Asthma increases pulmonary thromboembolism risk: a nationwide population cohort study. Eur Respir J 2014; 43: 801-807.

90 American Thoracic Society, European Respiratory Society. American Thoracic Society/European Respiratory Society International Multidisciplinary Consensus Classification of the Idiopathic Interstitial Pneumonias. Am J Respir Crit Care Med 2002; 165: 277-304.

91 Crooks MG, Hart SP. Coagulation and anticoagulation in idiopathic pulmonary fibrosis. Eur Respir Rev 2015; 24: 392-399.

92 Scotton CJ, Krupiczojc MA, Königshoff M, et al. Increased local expression of coagulation factor X contributes to the fibrotic response in human and murine lung injury. J Clin Invest 2009; 119: 2550-2563.

93 Bastarache JA, Wang L, Geiser T, et al. The alveolar epithelium can initiate the extrinsic coagulation cascade through expression of tissue factor. Thorax 2007; 62: 608-616.

94 Mostefai HA, Meziani F, Mastronardi ML, et al. Circulating microparticles from patients with septic shock exert protective role in vascular function. Am J Respir Crit Care Med 2008; 178: 1148-1155.

95 Buesing KL, Densmore JC, Kaul S, et al. Endothelial microparticles induce inflammation in acute lung injury. J Surg Res 2011; 166: 32-39.

96 Bucciarelli P, Martinelli I, Artoni A, et al. Circulating microparticles and risk of venous thromboembolism. Thromb Res 2012; 129: 591-597.

97 Garcia Rodriguez P, Eikenboom HC, Tesselaar ME, et al. Plasma levels of microparticle-associated tissue factor activity in patients with clinically suspected pulmonary embolism. Thromb Res 2010; 126: 345-349.

98 Thaler J, Koppensteiner R, Pabinger I, et al. Microparticle-associated tissue factor activity in patients with acute unprovoked deep vein thrombosis and during the course of one year. Thromb Res 2014; 134: 1093-1096.

99 Connolly GC, Francis CW. Cancer-associated thrombosis. Hematology Am Soc Hematol Educ Program 2013; 2013: 684-691.

100 Siegel R, Ward E, Brawley O, et al. Cancer statistics, 2011: the impact of eliminating socioeconomic and racial disparities on premature cancer deaths. CA Cancer J Clin 2011; 61: 212-236.

101 Galiè N, Hoeper MM, Humbert M, et al. Guidelines for the diagnosis and treatment of pulmonary hypertension. Eur Heart J 2009; 30: 2493-2537.

102 Archer S, Rich S. Primary pulmonary hypertension: a vascular biology and translational research "Work in progress". Circulation 2000; 102: 2781-2791.

103 Farber HW, Loscalzo J. Pulmonary arterial hypertension. N Engl J Med 2004; 351: 1655-1665.

104 Budhiraja R, Tuder RM, Hassoun PM. Endothelial dysfunction in pulmonary hypertension. Circulation 2004; 109: 159-165.

105 Humbert M, Morrell NW, Archer SL, et al. Cellular and molecular pathobiology of pulmonary arterial hypertension. J Am Coll Cardiol 2004; 43: Suppl. 12, 13S-24S.

106 Rabinovitch M. Molecular pathogenesis of pulmonary arterial hypertension. J Clin Invest 2012; 122: 4306-4313.

107 Tual-Chalot S, Guibert C, Muller B, et al. Circulating microparticles from pulmonary hypertensive rats induce endothelial dysfunction. Am J Respir Crit Care Med 2010; 182: 261-268.

108 Pfister SL. Role of platelet microparticles in the production of thromboxane by rabbit pulmonary artery. Hypertension 2004; 43: 428-433.

109 Aliotta JM, Pereira M, Amaral A, et al. Induction of pulmonary hypertensive changes by extracellular vesicles from monocrotaline-treated mice. Cardiovasc Res 2013; 100: 354-362.

110 Visovatti SH, Hyman MC, Bouis D, et al. Increased CD39 nucleotidase activity on microparticles from patients with idiopathic pulmonary arterial hypertension. PLoS One 2012; 7: e40829.

111 Diehl P, Aleker M, Helbing T, et al. Increased platelet, leukocyte and endothelial microparticles predict enhanced coagulation and vascular inflammation in pulmonary hypertension. J Thromb Thrombolysis 2011; 31: 173-179.

112 Nadaud S, Poirier O, Girerd B, et al. Small platelet microparticle levels are increased in pulmonary arterial hypertension. Eur J Clin Invest 2013; 43: 64-71. 